\title{
Conscience Ecologique Et Developpement Durable: Constat Et Défis
}

\author{
EHOUMA Koffi Ludovic, Assistant, Enseignant-chercheur \\ N'GUESSAN Kouamé Hendersonn, Assistant, Enseignant- \\ chercheur \\ Université Félix HOUPHOUËT-BOIGNY de Cocody, Côte d'Ivoire
}

doi: 10.19044/esj.2016.v12n29p232 URL:http://dx.doi.org/10.19044/esj.2016.v12n29p232

\begin{abstract}
The efficient environmental management is a real problem for sustainable development in our countries. From observations, it appears that the destruction of environmental cover is linked to economic growth and a backward thinking that harm the environmental health. To remedy this, an awareness and a profound reform based on an ecological education is needed. This reflection aims at making a contribution to the understanding and promotion of a real policy on environmental health following the development by Edgar Morin.
\end{abstract}

Keywords: Environmental health, environmental awareness, sustainable development

\section{Résumé}

La gestion efficiente de l'environnement pose un véritable problème pour le développement durable dans nos Etats. A partir observations faites à travers une revue documentaire, il ressort que la destruction de l'environnement est liée à la croissance économique et aux mentalités rétrogrades qui mettent en mal la santé environnementale. Pour y remédier, une prise de conscience et une reforme profonde basées sur une véritable éducation écologique s'imposent. Cette réflexion vise à apporter une contribution à la compréhension et à la promotion d'une véritable politique de santé environnementale suivant le développement selon Edgar Morin.

Mots clés: Santé environnementale, conscience écologique, développement durable 


\section{Introduction}

De profonds écarts de développement s'observent à toutes les échelles : entre les États développés et les États en développement, mais aussi à l'intérieur de ces États. Le développement des pays du Sud, à l'image de celui des pays du Nord, menace la santé de l'environnement. Cette situation exige de trouver des modes de croissance plus équitables, qui gaspillent moins les ressources de l'environnement, et d'inventer un développement durable.

La question de départ de cette réflexion stipule qu'en ce début du XXIème siècle, qu'est ce qui peut être identifié comme l'événement clé, le problème, de portée globale et locale ?

Les prémisses de réponse à cette question interrogent la destruction du potentiel démocratique, la pauvreté et la faim à une échelle massive, le déficit croissant de santé et d'éducation, l'explosion des conflits et des violences, la disparité persistante entre les régions, entre les hommes et les femmes, entre les filles et les garçons ou encore la négation des droits humains, etc. soit encore, pour rassembler dans un seul concept ces réponses, le déficit de Développement ?

La réponse est oui, pour l'Afrique subsaharienne, le déficit de Développement, serait le problème clé. Mais, voilà qu'une autre question surgit avec cette réponse.

Dans son rapport à l'Environnement (écosystème, biodiversité, biotope, biocénose...) le Développement se révèle à nous aujourd'hui comme une «idée sous-développée », pour utiliser l'expression d'Edgar MORIN (2011). La chose est préoccupante, car d'un côté, nous avons l'émergence de la «conscience écologique » portée par une science nouvelle, une philosophie nouvelle, une théologie nouvelle, culture de défense d'un nouvel humanisme. De l'autre, c'est le Développement, pour utiliser la formule d'Edgard MORIN (2001) qui «refuse à l'humanité d'accéder à l'humanité »).

$\mathrm{Au}$ plan de l'histoire des idéologies et des pratiques du développement, l'apparition (en 1987) du concept de «développement durable » traduirait un progrès, en ce qu'il intègre trois dimensions, l'économie, l'écologie et le social: " à long terme, il n'y aura pas de développement possible s'il n'est pas économiquement efficace, socialement équitable et écologiquement tolérable " Rapport sur le développement durable, 2014). En somme, une forme de développement économique respectueux de l'environnement, du renouvellement des ressources et de leur exploitation rationnelle, de manière à préserver les matières premières, un mode de développement qui répond aux besoins du présent sans compromettre la capacité des générations futures à répondre à leurs propres 
besoins (Rapport Brundtland, 1987). La conférence de Rio (1992) en fait un paradigme du développement pour la communauté internationale.

Le constat est que l'on observe un écart entre le paradigme du développement durable et la rationalité et les pratiques dominantes actuelles du Développement. En d'autres termes, le développement durable est resté une idée, rien qu'une idée. Le développement n'est pas toujours respectueux de l'environnement, il n'est pas écologiquement tolérable. Il y a là un problème pour la réflexion, la recherche : comprendre, rendre intelligible cette contradiction. Ainsi, pourquoi le « développement durable » est-il resté un projet impossible.

L'objectif de cette réflexion est de suggérer un schème de compréhension de cette antinomie qui caractérise les sociétés de la modernité, et d'identifier en conséquence, les vrais enjeux de la santé environnementale pour notre temps, en tous lieux, en Afrique comme ailleurs.

La problématique de la santé environnementale, ce sont les humains qui subissent la situation agonique de ce début de millénaire. Tous les humains partagent un destin de perdition. L'Organisation mondiale de la santé souligne ce fait lors de la Conférence ministérielle santé et environnement: "L'environnement est la clé d'une meilleure santé », en incluant dans cette problématique des paramètres liés à la qualité des milieux (pollution de l'atmosphère, de l'eau, des sols, déchets mais aussi nuisances sonores, insalubrité, etc.) et à l'ensemble des activités humaines (air ambiant, accidents domestiques, violences urbaines, etc.) (OMS, 1999).

C'est le lieu de déconstruction des mythes de la trilogie: mondialisation, occidentalisation, développement. L'examen de cette problématique à partir d'une analyse discursive par une revue documentaire de portée globale et locale, articule: une Thèse, des Observations et des enjeux de la conscience écologique.

\section{La these}

Une conjonction de menaces contre la santé environnementale et l'homme, se présente en ce début du millénaire. Le facteur en cause est l'environnement social ou le milieu humain voire la civilisation. L'environnement social, est identifié ici, comme ce "processus déchaîné aux trois faces mondialisation, occidentalisation, développement qui dégrade la biosphère de façon irrésistible, aussi bien globalement que localement » (E. MORIN, 2011)

L'analyse sociologique caractérise encore cet environnement social aux trois faces : mondialisation, occidentalisation et développement, comme une dynamique qui peut être assimilée à un "déchaînement désintégrateur" qui ne trouve pas ses contrôles et ses régulateurs, et surtout, 
le système qui le produit, l'organise, ne connaît qu'une dynamique cyclique répétitive, à l'épreuve des crises (dont la crise de la santé environnementale), soit encore une dynamique incapable de récurrence créatrice, de métamorphose, comme si son histoire épuise toutes les possibilités historiques.

\section{Les observations}

L'analyse des observations qui illustre la thèse s'appuie sur deux axes :

- le système désintégrateur de l'environnement social

- les racines mentales du système désintégrateur

\section{Le systeme desintegrateur de l'environnement social}

Les vecteurs de la croissance économique passent par la multiplication des pollutions urbaines, pollutions agricoles, pollutions atmosphériques, de rivières, de lacs, de mers (multiples marées noires, les deux dernières en date dans le golfe du Mexique et en Chine), dégradant des sols et des nappes phréatiques, favorisant les catastrophes nucléaires (Tchernobyl, Fukushima), et les déchets toxiques des technologies du nucléaire, le réchauffement climatique, avec les déforestations massives, les effets polluants des énergies fossiles et des urbanisations sauvages...

Les catastrophes naturelles sont du quotidien des populations, multiples, multiformes et en très grande progression. Il ressort qu'en « 2011, 302 désastres naturels ont touché la planète. Ces aléas ont provoqué la mort de 29.782 personnes, affecté 260 millions d'êtres humains et entrainé des dégâts de l'ordre de 286 milliards d'euros. Pire, la moyenne des sinistres est en hausse de 30\% depuis 2010. C'est ainsi que la Banque mondiale a identifié 86 pays à haut risque de tremblements de terre, de cyclones, d'inondations, de sécheresse, parmi lesquels la Côte d'Ivoire. Le pays est vulnérable surtout face aux changements climatiques dont les impacts consistent en une érosion côtière, des inondations fréquentes, des glissements de terrains, l'émergence de certaines maladies, une perte de la biodiversité. Aussi, des catastrophes directement provoquées par l'homme avec des conséquences environnementales importantes comme les feux de brousse, les déversements d'hydrocarbures en mer (mars 2006 au large de Jacqueville), le déversement des déchets toxiques (dans la nuit du 19 au 20 août 2006, le cargo Probo Koala dispersait plus de 500.000 litres de résidus toxiques à Abidjan, provoquant 17 morts et des milliers d'intoxications en $2006 »$ (L'intelligent d'Abidjan, 25 avril 2013)

Sont ainsi affectées, toutes les facettes de la vie humaine et naturelle, urbaines, rurales, aquatiques, océaniques (notamment la destruction des 
réserves de pêche). Ce dynamisme détruit la biodiversité et altère la diversité culturelle. Les très grands progrès en hygiène et médecine sont entachés par de très grands accroissements des multiples pollutions et des sources de stress agissant sur la santé, fragilisant l'espèce humaine.

Le capital financier mondial, dont le dynamisme coproduit la globalisation, aggrave la santé écologique. Il conduit à la surexploitation des ressources naturelles partout dans le monde, surtout dans l'hémisphère Sud ; il a incité à multiplier les "externalités », dommages collatéraux écologiques, dont le déversement de déchets toxiques dans les pays pauvres (déversement des déchets toxiques août 2006 à Abidjan); il a stimulé ces pays à orienter leur agriculture vers l'exportation au détriment de leur agriculture vivrière, de leurs forêts, de leur biodiversité.

La raréfaction des ressources naturelles a aiguillonné l'esprit spéculatif, d'où les hausses de prix qui ont accru les ménages pauvres du monde riche, lesquels se sont surendettés sous les incitations du même capitalisme financier.

\section{Les racines mentales du systeme desintegrateur}

La Côte d'Ivoire vit avec le développement de la civilisation occidentale, sous l'emprise d'une pensée disjonctive (qui sépare ce qui est inséparable) et d'une pensée réductrice (qui réduit le complexe au simple). Il nous faut être attentif à cette disjonction absolue entre l'humain et le naturel qui a résulté de deux matrices culturelles.

La première est relative à la prégnance de l'anthropocentrisme judéochrétien. Nos observations montrent que culturellement, le judéochristianisme fait l'objet d'une accusation récurrente. Il est la racine d'un anthropocentrisme réducteur à l'interne, pour soi, et dominateur à l'externe, quant à la nature. La tradition ou la matrice judéo-chrétienne est donc en procès. L'anthropocentrisme se révèle comme le chef d'accusation central. L'homme est au centre, tout s'y ramène, et il ramène tout à lui, et la nature se trouve neutralisée, extériorité stricte, offerte à maîtrise, voire à manipulation. Il ressort que ce procès a bel et bien entretenu, voire nourri, consciemment ou non, un anthropocentrisme réducteur et dangereux. L'humain est exception à la nature. Il n'existe qu'à s'en distinguer, à la tenir à distance et même à y inscrire des différences et des séparations qui en font un cadre de vie possible, pas plus, et non un ensemble indifférencié, du coup menaçant et envahissant. Alors que la plupart des religions dont l'hindouisme, le bouddhisme et les religions traditionnelles d'Afrique noire, immergent l'humain dans le cycle des reproductions du monde vivant. Le monothéisme juif, puis chrétien et islamique, a disjoint l'homme du monde animal, de la nature. 
La deuxième matrice désintégratrice est l'essor et la globalisation du rationalisme occidental où les ressources environnementales ont toujours constitué le socle des activités humaines notamment en termes de production et de consommation. Pour Descartes (1637), l'homme est l'unique sujet dans l'univers, le seul à posséder une âme, et il a vocation à devenir «comme maître et possesseur de la nature ». L'homme adopte ainsi une attitude de dominance sur l'ordre naturel. Le développement technique, économique, capitaliste de la civilisation occidentale entreprend dès lors la conquête de cette nature où tout ce qui est vivant est objet à asservir, manipuler ou détruire.

\section{Enjeux de la conscience ecologique}

La rupture avec ce système désintégrateur peut être appréhendée, comme un passage de la chimère de la trilogie -mondialisation, occidentalisation, développement- à la reforme, reforme de civilisation, reforme de société, reforme de vie. Cette épreuve de rupture, est la problématique de la santé écologique, dont nous esquissons quelques enjeux.

\section{La chimere}

Les progrès de la science, de la technique, de l'industrie se heurtent dans une ambivalence significative. Ils apportent non seulement des bienfaits que l'humanité n'a jamais connus, mais aussi les plus grandes menaces que l'humanité ait jamais connues, à la fois asservissement et libération, régression et progression, mieux-être et mal-être, vie et mort.

Depuis quelques années, des voix s'élèvent pour dénoncer les conséquences catastrophiques entraînées par le développement des technosciences et du modèle économique qui lui est lié. Ces voix multiplièrent les avertissements concernant les graves dérèglements climatiques provoqués par les activités humaines, la destruction de la biodiversité avec ses conséquences sur les équilibres planétaires, l'inanité d'un modèle de développement fondé sur une croissance illimitée, exponentielle, de la production industrielle dans une planète limitée, etc. Les ouvrages «Mal de terre» (Seuil, 2003) de Hubert REEVES et «Ces maladies créées par l'Homme », (Albin Michel, 2004) de Dominique BELPOMME, posent la question cruciale: "le sort de l'aventure humaine, entamée il y a des millions d'années, va-t-il se jouer en l'espace de quelques décennies? 》. Ce qu'Edgar Morin (2000) résume comme suit : «Depuis les années 70, nous avons découvert que les déjections, émanations, exhalaisons de notre développement technico-industriel urbain dégradent notre biosphère et menacent d'empoisonner irrémédiablement le milieu vivant dont nous 
faisons partie : la domination effrénée de la nature par la technique conduit l'humanité au suicide ».

Aussi, le mode de pensée actuel est-il fondé sur la disjonction absolue entre l'humain et le naturel. La compartimentation des disciplines et des catégories nous empêche de faire la relation entre les parties et le tout. La pensée occidentale ne sait opérer que par disjonction ou réduction. « La chimère de maîtrise totale $\mathrm{du}$ monde, encouragée par le prodigieux développement des sciences et techniques, se heurte aujourd'hui à la prise de conscience de notre dépendance à l'égard de la biosphère et à celle des pouvoirs destructeurs de la techno-science pour l'humanité elle-même ») (E. MORIN, 2011).

\section{La reforme attendue}

\section{La réforme de la pensée}

Les voies pour répondre à la menace écologique ne sont pas seulement techniques, elles nécessitent prioritairement une réforme de notre mode de pensée (inséparable d'une réforme du système de l'éducation, du système de la connaissance) pour embrasser dans sa complexité la relation entre l'humanité et la nature, et concevoir des reformes de civilisation, des reformes de société, des reformes de vie.

Dans le dernier tiers du XXè siècle est née la première science systémique transdisciplinaire, l'écologie. Elle est contemporaine des progrès de la cosmologie, des sciences de la terre, de la préhistoire, lesquelles ont développé une connaissance scientifique en dépassement du rationalisme qui réduit le complexe au simple, et de la pensée théologique et philosophique qui sépare l'humain de la nature.

Cet attentisme ou cette absence de réforme institutionnelle de la pensée par l'éducation, la connaissance avec les formidables acquis de l'écologie, science d'un type nouveau, révélant notre relation de vie et de mort avec la biosphère, nous oblige à repenser notre planète, à y lier notre destin et finalement, à nous repenser nous-mêmes.

D'où le problème fondamental: notre «conscience écologique » émerge, localement et globalement, par sauts, à l'occasion de dégâts, dégradations et catastrophes. Et cette émergence ne progresse pas, elle est freinée par la résistance des structures institutionnelles et mentales sclérosées, par d'énormes intérêts économiques. Ainsi, de nombreux verrous subsistent en dépit des grandes conférences internationales qui ont jalonné ces dernières décennies. Pour résumer, l'épanouissement de la « conscience écologique » est bloqué, elle ne connaît pas d'incarnation politique, économique, sociale.

Edgar MORIN identifie cette situation, en ce début du millénaire : « le refus fait à l'humanité d'accéder à l'humanité ». 
Suivant André MALRAUX, figure de l'humanisme, nous devons aller à la culture authentique, celle qui «cherche à découvrir l'homme, partout où elle découvre ce qui écrase l'homme ». Nous pouvons alors comprendre la tendance des mouvements écologistes à la rencontre des cultures, la religion et l'art, notamment.

\section{La rencontre de l'écologie et de la religion}

Initialisée en 1986, à la rencontre d'Assise, c'est une tendance large et profonde des mouvements écologistes de mobiliser les ressources de spiritualité et de culture religieuse (les visions et conceptions du monde, l'ouverture à la transcendance) pour surmonter au lieu de subir l'apocalypse écologique planétaire que construit sous nos yeux le système désintégrateur de l'environnement social. De son côté, la théologie (chrétienne, islamique, juive) a revisité les textes, éclairant l'exigence écologique au cœur des textes sacrés. Lama Denys (2004) souligne le champ de combat de la théologie bouddhiste: «nos sociétés de consommation sont des sociétés de consumation, il suffit de voir le désastre écologique. Que faire ? Parce que les vampires préfèrent l'obscurité, nous dévons mettre en lumière leur activité, car l'éclairage les effraie. »

\section{L'appel à l'art, au grand art}

L'écologisme, l'humanisme écologiste, en quête de réforme de civilisation, de réforme de vie, ne peut ignorer le formidable potentiel libérateur de l'art, du grand art, à la fois «près et loin de la vie sociale ». André MALRAUX (1951) nous montre comment le grand art (peinture, théâtre, musique, poésie, sculpture) se noue en sens inverse du courant social. Il est le contrepoint, le «chant» de l'histoire. "Quelque fois lié à la civilisation où il naît, l'art la déborde souvent, la transcende... comme s'il faisait appel à des pouvoirs qu'elle ignore, à une inaccessible totalité de l'homme... Chacun des chefs d'œuvre est une purification du monde, et la victoire de chaque artiste sur sa servitude, rejoint, dans un immense déploiement, celle de l'art sur le destin de l'humanité. L'art est un antidestin»».

\section{Conclusion}

L'idée généralement admise de la trilogie (mondialisation, occidentalisation, développement) porteuse de la « Civilisation », est aveugle aux dégâts et dégradations écologiques qu'elle continue de provoquer. L'observation est catastrophique : cette «civilisation », cet environnement social sans «contrôles et sans régulateurs » se développe dangereusement, comme un dynamisme désintégrateur du milieu du vivant, de la biosphère. 
D’un côté, nous avons l'émergence de la « conscience écologique » portée par une science nouvelle, une philosophie nouvelle, une théologie nouvelle, culture de défense d'un nouvel humanisme. De l'autre, c'est le Développement, pour utiliser la formule d'Edgard MORIN qui « refuse à l'humanité d'accéder à l'humanité ».

Devant la gravité de la situation pour l'avenir de Homos sapiens, on mesure la portée de la Déclaration (Juin 2002) de Jean PAUL II, le Pape qui a proclamé François d'Assise patron des écologistes. "L'écologie n'est pas une mode, un courant politique ; elle est une exigence éthique, un impératif moral qui presse l'humanité à assumer ses propres responsabilités. La fierté de notre époque est d'avoir éveillé et développé une conscience écologique. " Comme la voie suivie par l'humanité conduit à l'aggravation de tous les maux et périls, il s'agit, par prises de conscience et par reformes, de changer de voie. "Homo sapiens" ne doit plus chercher à dominer la Terre, mais à la ménager et à l'aménager.

Nous retenons des analyses : si les défis de la conscience écologique pour une santé écologique débordent de loin la culture, il n'y a sans celle-ci, la culture authentique, aucune chance de les relever.

\section{References:}

1. COMMISSION MONDIALE SUR L'ENVIRONNEMENT ET LE DÉVELOPPEMENT / ONU (1987): Rapport Brundtland, notre avenir à tous. Oxford University Press.

2. DENYS L., Le « Forum écologie et spiritualité » de 2004, Le Monde des Religions, $\quad n^{\circ} 39, \quad 2010$, http://www.passerelleco.info/article.php?id_article=334, consulté le 21 janvier 2016.

3. DESCARTES R. (1637), Discours de la méthode, 6 ème partie, Bibliothèque de la Pléiade, Éd. Gallimard, 1966.

4. MALRAUX A. (1951), Les Voix du silence, dans Écrits sur l'art I, Gallimard, Bibliothèque de la Pléiade, p. 897

5. MORIN E. (1982), Science avec conscience, Fayard, Nouvelle édition remaniée, coll. Points, 1990

6. MORIN E. (2000) : les sept savoirs nécessaires à l'éducation du futur. Paris, édition du Seuil.

7. MORIN E. (2001), L'identité humaine. Paris, édition du Seuil.

8. MORIN E. (2011) Conversation pour l'avenir (avec Gilles et Michel Vanderpooten), La Tour d'Aigues, L'Aube, coll. « Monde en Cours ».

9. MORIN E. (2011), Dialogue sur la connaissance : Entretiens avec des lycéens, Éditions de l'Aube.

10. MORIN E. (2011), Le chemin de l'espérance, en collaboration avec Stéphane Hessel, Fayard 
11. ORGANISATION MONDIALE DE LA SANTE, 1999, Conférence ministérielle Santé et environnement http://inpes.santepubliquefrance.fr/10000/themes/sant e environnement/index.asp, consulté le 21 janvier 2016.

12. RAPPORT ANNUEL DE DÉVELOPPEMENT DURABLE (2014) www.corse.fr/attachment/550965 · Fichier PDF, consulté le 21 janvier 2016.

13. TODD O., MALRAUX A. (2001), une vie, éd. Gallimard. 